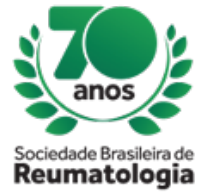

\title{
RITUXIMAB IN REFRACTORY SJOGREN SYNDROME - EXPERIENCE IN TERTIARY HOSPITAL
}

Laís Farrapo de Barros Leite (HSPE - IAMSPE, São Paulo, SP, Brasil), André Ozela Augusto (HSPE IAMSPE , São Paulo, SP, Brasil), Natalia Carneiro dos Santos (HSPE - IAMSPE, São Paulo, SP, Brasil), Sandra Echazú Roman (HSPE - IAMSPE, São Paulo, SP, Brasil), Elisa Pereira Vicentini (HSPE - IAMSPE, São Paulo, SP, Brasil), Estêvão da Costa Santana (HSPE - IAMSPE , São Paulo, SP, Brasil), Roberta de Almeida Pernambuco (HSPE - IAMSPE , São Paulo, SP, Brasil), Rina Dalva Neubarth Giorgi (HSPE - IAMSPE , São Paulo, SP, Brasil)

\section{BACKGROUND}

Sjogren's syndrome is mostly characterized by symptoms of fatigue, xerostomia and xerophthalmia, due to the dysfunction of the exocrine glands, which present periductal mononuclear infiltration, mainly by CD4 + T cells and B cells.

Severe systemic manifestations involve cutaneous involvement in the form of vasculitis; pulmonary, with the most common pattern of lymphoid interstitial pneumonia; renal, such as tubulointerstitial nephritis or glomerulonephritis. There may be involvement of the central and peripheral nervous system, with mixed polyneuropathies being the most frequent forms, in addition to hematological manifestations, characterized by cytopenias. The syndrome is notoriously associated with an increased risk of lymphoma.

Systemic forms require targeted therapy, often in need of conventional immunosuppressants, such as Methotrexate, Azathioprine, and Cyclosporine. However, in patients that are refractory to these therapies, it has been used immunobiological agents, and among them, Rituximab (RTX), a chimeric monoclonal antibody that hits CD20 on the surface of B cells, was the one that presented the greatest benefit.

\section{MATERIALS AND METHODS}

Data were collected from all patients with Sjogren's Syndrome from the Rheumatology outpatient clinic of a reference tertiary hospital in São Paulo and the indication and response to Rituximab was evaluated.

\section{RESULTS}

Eighty-eight patients were tabulated, all of them female. Among them, 10 were treated with Rituximab due to severe systemic manifestations, refractory to conventional treatment. A patient with transverse myelitis had almost complete remission of symptoms. Two patients used the drug because of the peripheral polyneuropathy, one of them had complete response and the other had partial response. A patient with segmental and focal glomerulosclerosis obtained complete and sustained remission of renal damage. Three patients had indication due to vasculitis, one of them presented infusional allergic reaction, so it was not possible to maintain the drug, and the others presented complete resolution of the manifestations. Two patients with coarse and refractory ulcerative keratitis used RTX, one had partial response and the other had total response of the ocular picture. One patient with interstitial lung disease was $100 \%$ successful with the indication of Rituximab.

\section{CONCLUSION}

The results confirmed the beneficial effects and the safety of Rituximab in the treatment of systemic manifestations of Sjogren's Syndrome in patients that are refractory to conventional drugs.

In our casuistry there was complete or partial response in 9 patients and only 1 failure. 
The present study ratifies what has been evidenced in the current literature, with Rituximab therapy promising in cases of Sjogren's syndrome refractory to conventional immunosuppressants. 